\title{
Roof cooling by direct evaporation from a porous roof layer
}

Salomé M.S. Kuehni (1), Elie Bou-Zeid (2), Colin Webb (1), Nima Shokri* (1)

(1) School of Chemical Engineering and Analytical Science, University of Manchester,

\author{
Manchester, M13 9PL, UK
}

(2) Department of Civil and Environmental Engineering, Princeton University, Princeton, NJ 08544, United States

*Corresponding author

Dr. Nima Shokri

School of Chemical Engineering and Analytical Science

Room C26, The Mill

The University of Manchester

Sackville Street, Manchester, M13 9PL, UK

Tel: 0441613063980

Email: nima.shokri@manchester.ac.uk

Group website: http://personalpages.manchester.ac.uk/staff/nima.shokri/ 


\section{Abstract}

As the world continues to urbanise, significant challenges are arising to environment, energy and water sustainability in cities. One of the most challenging consequences of increased urbanisation is increased energy consumption adversely affecting the quality of life, environment and public health. This motivated many researchers to find innovative methods to reduce energy consumption in buildings for cooling practices. In this paper, a series of experiments was conducted to investigate the performance of an evaporative layer of porous media and the effects of its particle size on reducing the roof surface temperature. To do so, customised rectangular Plexiglas columns were packed with three types of sand with welldefined particle size distribution saturated with water with all boundaries closed except the top, which was exposed to air for evaporation. The obtained results revealed the great potential of drying porous media to reduce the heat flux through roof via utilising a part of the energy for liquid vaporisation. As particle size decreased the temperature of roof remained lower than the bare roof for a longer time as a result of the presence of more liquid pathways connecting the receding drying front to the evaporation surface, which kept the surface wet for a longer time. Our results present new insights about the physical mechanisms controlling the performance of drying porous media to regulate roof surface temperature. 


\section{Introduction}

Increasing energy efficiency is an immediate priority to cut carbon emissions, secure energy as well as save on energy bills. Over the past few decades, the improvement of living standards and the affordability of air conditioning have led to a considerable increase in the energy consumption related to space cooling. Additionally, the so-called urban heat island effect, resulting from increased urbanisation, contributes to making cities several degrees hotter than their rural surroundings [1-3]. This increases energy consumption for cooling of residential and commercial buildings. Therefore, developing novel approaches to improve cooling energy efficiency of buildings is essential to meet our low carbon economy targets. This will become even more important in the near future with the increase in climate change, not only in hot, but also in temperate climates such as in the UK $[4,5]$.

Traditionally, roofs in some mid and high latitude locations consist of envelope materials (such as clay and asphalt) with a relatively high absorption coefficient, transferring large amounts of heat to the building. This leads to warmer indoor spaces in the summer and as a result higher demands for air conditioning. Roofs can represent up to $32 \%$ of the horizontal surface of built-up areas [6] and are important determinants of energy flux and heat transfer through the building envelopes. The roof offers the greatest opportunities for improving the cooling of buildings. It is the building element that is most exposed to the sky. Therefore, significant efforts have been made to reduce this heat load by improving roof design $[7,8]$. As a result of such efforts, several methods have been proposed. The so-called 'green roof' is an example of such a design $[9,10]$. A green roof is a roof that contains a soil (growing media) and vegetation layer as its outermost surface, allowing better regulation of building temperatures [9]. Although use of green roofs provides significant benefits, not only in energy consumption but also in urban microclimatological and environmental terms [11], 
some disadvantages have been noted as well. Installation cost is elevated and maintenance of the green roof system, i.e. watering and replacing the old plants, is required regularly at additional cost. Besides, in terms of building structure, enhanced structural support is required to accommodate green roof systems to ensure sufficient capacity to resist weight load under the soil and vegetation and avoid collapse [12], further adding to the cost.

Other examples of alternative roofs include roof ponds and evaporation-based roof cooling systems [7, 13]. While no porous medium is used in these evaporative roof systems, latent heat of evaporation remains the main mechanism used to cool the building roof [14], as with green roofs. Crawford and da Silva [13], among many others, studied the capability of a roofbased evaporative pumping system to lower thermal loadings imposed on the interior of a building to reduce energy consumption as a result of air conditioning. Jain [15] presented thermal models to evaluate the performance of various passive cooling roof systems including bare roof, wetted roof surface, and evaporative cooling using water pond with movable insulation and concluded that a roof pond with movable insulation offers an efficient method for roof cooling.

There are many papers that investigated evaporation-based (green or otherwise) roof cooling systems and a comprehensive review is beyond the scope of the present paper. But in general, the effectiveness and viability of these types of roofs depend very strongly on the local settings such as local climate, native vegetation, and water availability $[1,16,17]$. It should be also noted that the very concepts of these types of roofs are designed for regions with abundant rainfall and water resources, and with native vegetation species that produce high cooling by evapotranspiration (in the case of green roofs). Considering the rising temperature trends even in temperate cities (e.g. London), it is important to develop innovative, 
sustainable and cost-effective approaches capable of cooling buildings under a variety of conditions.

Recently, there have been some efforts to evaluate the performance of porous materials to be used on roofs (without vegetation) to create an effective system for reducing heat flux through buildings by utilizing their moisture absorption and evaporation capabilities [12]. A robust explanation and quantitative tool to predict the performance of a given drying porous media on reducing the heat flux through buildings are still missing due to a lack of deep physical understanding of how the thermal performance of drying porous media is affected by their transport properties, the properties of the evaporating fluid, and the external boundary conditions [18-22].

Typically the evaporation rate from initially saturated porous media is relatively high and is controlled by the atmospheric condition, the so-called stage-1 evaporation. During this period, liquid water is transported toward the evaporation surface via the capillary induced liquid flow connecting a receding drying front, marking the interface between the saturated and unsaturated zone [23], to the evaporation surface where liquid vaporization takes place supporting the evaporative demand. At a certain drying front depth or surface water content, the liquid continuity between the drying front and the evaporation surface is ruptured as a result of the interplay among the upward capillary force and the downward gravity and viscosity forces. Consequently the liquid meniscus recedes from the surface to a level below the surface forming an overlying thin dry layer. This marks the end of stage- 1 evaporation [24]. When all liquid meniscuses are disrupted, a new vaporization plane forms very close to the surface which marks the onset of the so-called stage- 2 evaporation. During this period, liquid is transported from the saturated zone to the new vaporization plane formed inside the porous medium followed by liquid vaporization at that level and vapour diffusion through the 
overlying dry layer [25]. Stage-1 and stage-2 evaporation are limited by the atmospheric conditions and the transport properties of porous media, respectively [26-28].

In the present paper, a novel approach was proposed to reduce the energy consumed for cooling buildings by utilizing drying porous materials (with no vegetation) on roofs. By consuming a part of the radiative energy received at the roof surface for liquid vaporization, the evaporative porous layer reduces the heat flux through the roof, as well as the convective heating of the outdoor space that results in the urban heat island effect [29]. The main appeal of this approach compared to green roofs is that brackish or salt water can be used for evaporation (it can thus be used in dry coastal areas), no maintenance of vegetation is required, and the roof depth can be quite small since it does not need to support a root layer (posing lesser constraints on the built structure). Therefore, motivated by the importance of developing low-energy methods for cooling buildings, the specific objectives of the present paper are a) to evaluate the application of drying porous media on moderating the temperature of irradiated surfaces under laboratory conditions and b) to investigate the effects of particle size on the performance of the drying porous media for this purpose. This paper is a first step in a larger effort aimed at developing such evaporative roofs.

\section{Experimental considerations}

All experiments were conducted in the Multiphase Flow and Porous Media research laboratory in the School of Chemical Engineering and Analytical Science at The University of Manchester. An experimental setup was developed to investigate the thermal performance of drying porous media, in terms of moderation of the roof surface temperature and reducing heat fluxes towards the indoor space. The experimental station is illustrated in Fig. 1. 


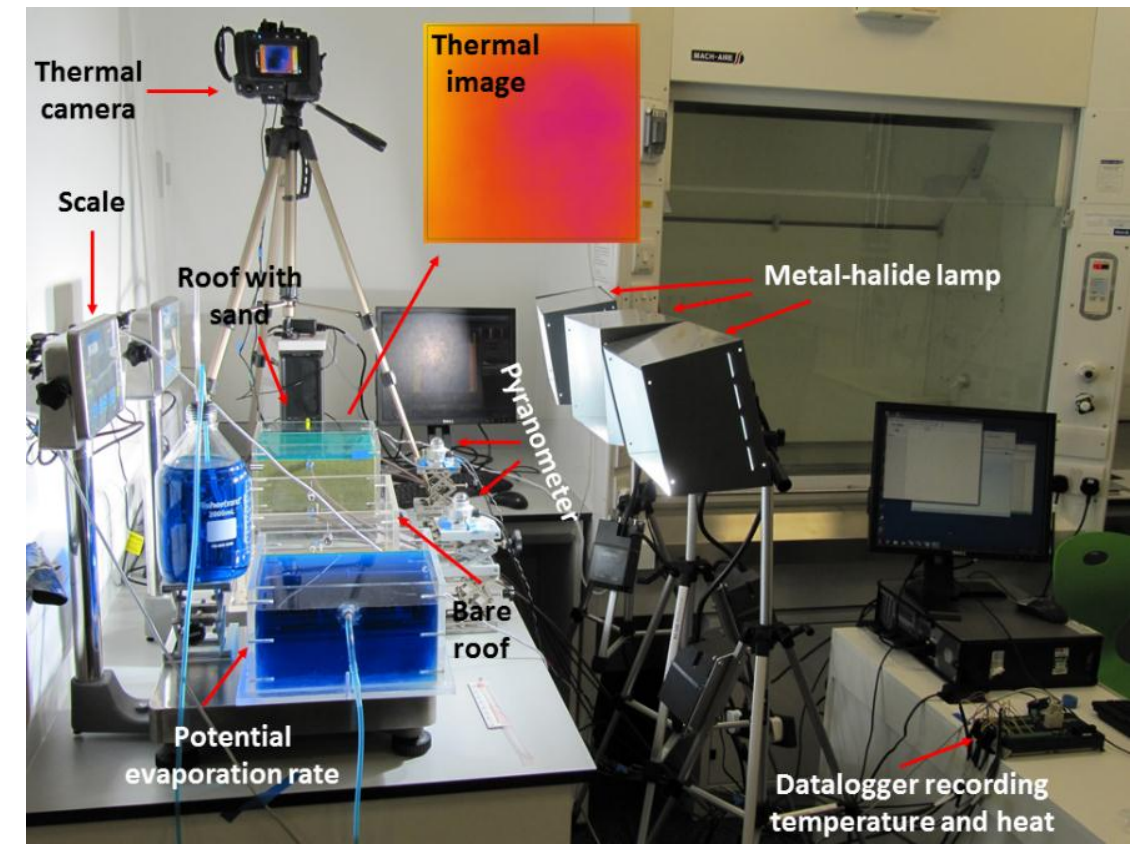

Fig. 1. Experimental setup. The container filled with dyed water was used to measure the potential evaporation rate. The container packed with sand particles (labelled as "Roof with sand") was used to investigate the performance of drying porous media in reducing the surface temperature compared to the bare roof (the middle column). A thermal camera was fixed on top of the roof covered with drying porous media. Metal halide lamps were used to heat the containers. Two pyranometers were used to measure the radiation flux (they were positioned parallel to the roofing surfaces to capture the flux normal to these surfaces). The column packed with sand grains was equipped with thermocouples at the surface and bottom of the column. Additionally, a heat flux sensor was buried in the middle of the sand column. The data measured by the thermocouples, heat flux sensors and the pyranometers were recorded using a datalogger connected to a PC.

Three samples of quartz sand differing in particle size distribution referred to as coarse, medium and fine sand were used to pack the customized Plexiglas containers $(150 \mathrm{~mm}$ in height, $250 \mathrm{~mm}$ in width and $250 \mathrm{~mm}$ in length). Fig. 2 shows the particle size distribution of the sand samples used in our experiments (provided by the manufacturer). 


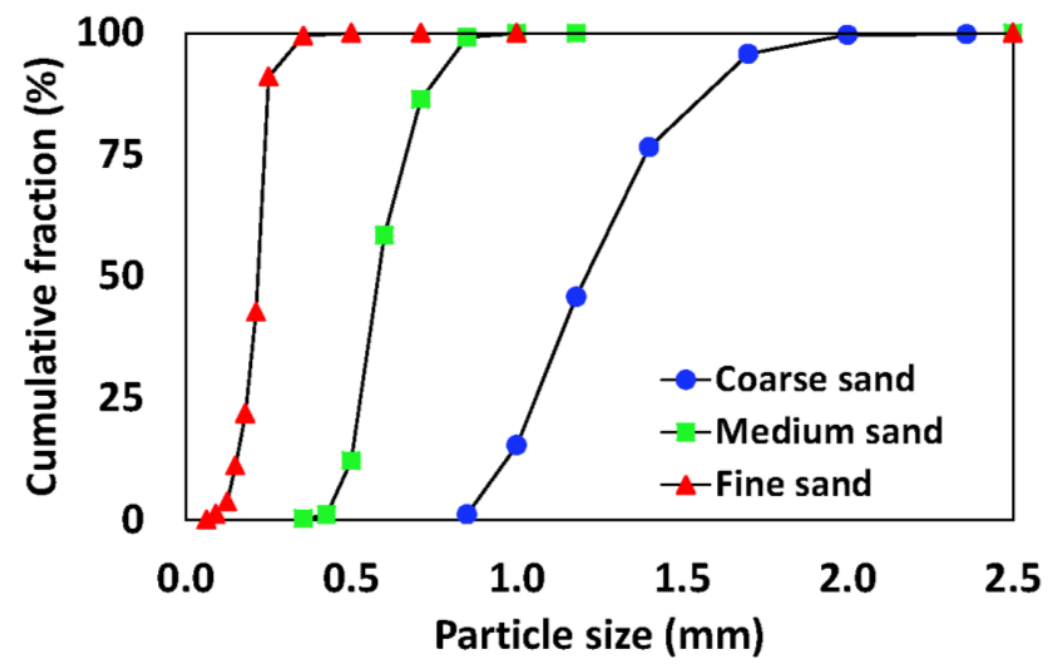

Fig. 2. Particle size distribution of different sands used in the experiments.

The container was packed with sand grains saturated with water following the procedure described in Grapsas and Shokri [30]. To measure the evaporation rate, the containers were mounted on digital balances (with accuracy of $0.1 \mathrm{~g}$ ) connected to a computer to record the mass every 5 minutes during the experiments. The container was closed from all boundaries except the top, which was exposed to air for evaporation. The container packed with sand grains was equipped with thermocouples specifically designed to measure soil temperature (105E Type E Thermocouple, Campbell Scientific) with the accuracy of $0.5^{\circ} \mathrm{C}$. The thermocouples were placed at the surface and bottom of the sand pack to measure the temperature at these two locations every 5 minutes during the course of the experiment. The data were recorded using a datalogger (CR1000, Campbell Scientific). In addition, a thermal camera (FLIR T650sc, FLIR Systems, Inc.) with a thermal sensitivity (at $30{ }^{\circ} \mathrm{C}$ ) of less than $30 \mathrm{mK}$ and a resolution of $640 \times 480$ pixels was fixed above the packed sand to record the dynamics of temperature evolution at the surface every 30 minutes. A square heat flux sensor (35.1 $\mathrm{mm}$ by $35.1 \mathrm{~mm}$ ) with a thickness of $2.8 \mathrm{~mm}$ (Wuntronic $\mathrm{GmbH}$, Germany) and accuracy of $5 \%$ connected to the datalogger was placed in the middle of the sand pack to measure the heat flux during the evaporation process every 5 minutes. 
In addition to the container packed with sand grains, in each round of the experiment, two more containers (with the same dimensions as mentioned above) were used to measure the potential evaporation rate and the temperature at the bare roof as illustrated in Fig. 1. A thermocouple was placed on the surface of the bare roof (covered by roofing felt) to record dynamics of the roof temperature during the experiment. To measure the potential evaporation rate, the $3^{\text {rd }}$ container was mounted on a digital balance (to record the mass every 5 minutes), filled with dyed water ( $3 \mathrm{~mL}$ per litre), and connected to a Mariotte siphon (also mounted on the same balance) that maintained the water surface in the container at a fixed height [31].

To simulate the solar radiation, three metal halide bulbs were used (EYE Color Arc PAR36, Iwasaki Electronic Co., Japan), which were fixed on customized stands facing toward the containers as illustrated in Fig. 1. Two pyranometers (Hukseflux, The Netherlands) were used to measure the shortwave radiation from the bulbs. The pyranometers were connected to the datalogger to record the data every 5 minutes during the course of the experiment. Special attention was given to establish equal radiation to the containers by adjusting the position and height of the solar lights until similar radiation data were recorded by the two pyranometers.

Using the described experimental station, three rounds of experiments were conducted with the fine, medium and course sand. The obtained results are explained next.

\section{Results and discussions}

\subsection{Evaporative mass losses}

The data measured by the balance were used to calculate the cumulative mass loss and the evaporation rate during each round of the experiment. Fig. 3 illustrates the obtained results. 

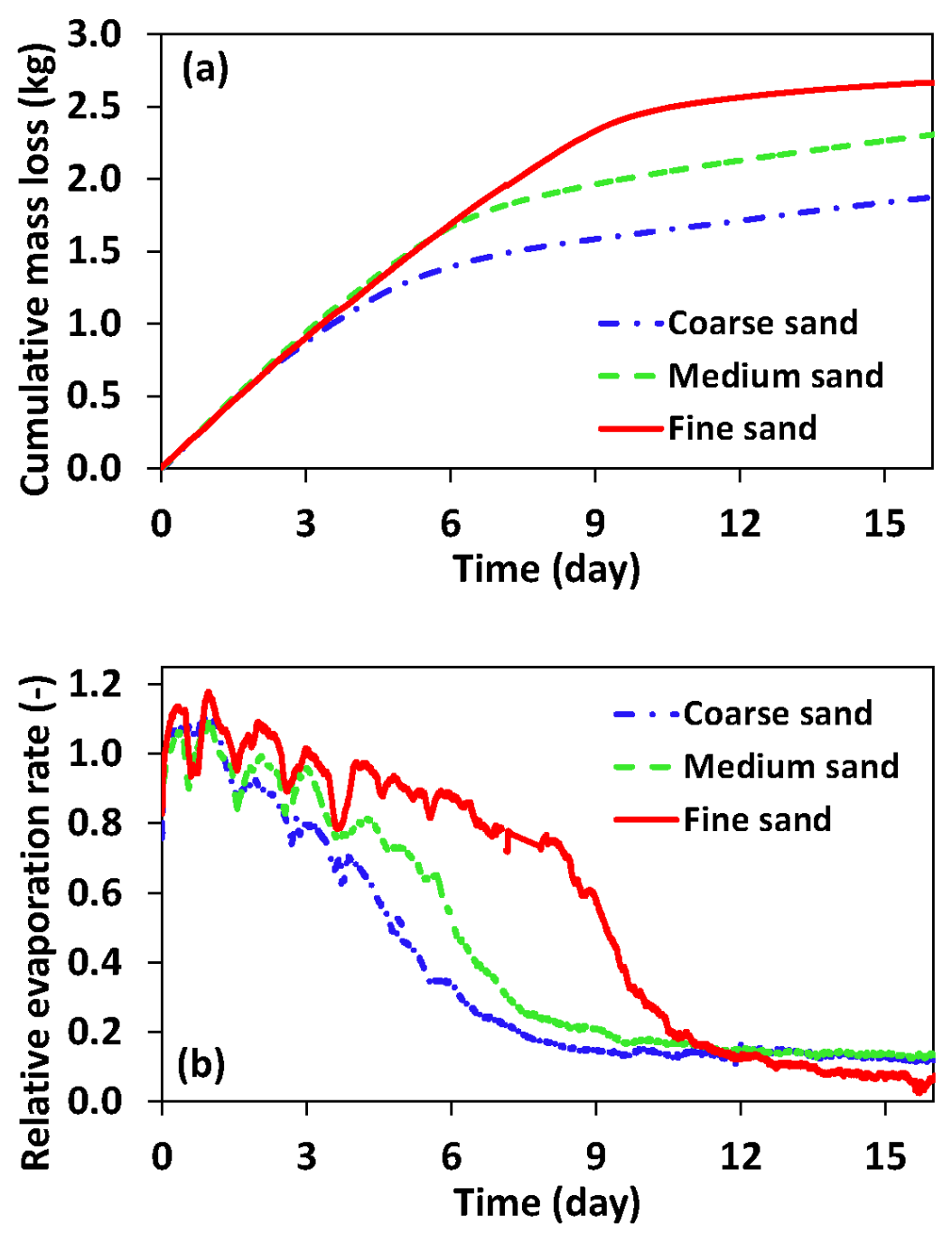

Fig. 3. (a) The measured cumulative evaporative losses during the experiments with the roofs filled with the coarse, medium and fine sand. The finer the particles, the longer the duration of stage-1 evaporation. (b) The evaporation rates measured during drying of the coarse, medium and fine sands placed over the roofs. Note that the drying rate was scaled by the measured potential evaporation rate from the container filled with water. This dimensionless rate is referred to as "Relative evaporation rate".

During the experiments, we did not control the air speed, ambient temperature, or relative humidity. The average ambient temperature during the experiments with the coarse, medium and fine sand was $27.8,29.4$ and $30.1{ }^{\circ} \mathrm{C}$, respectively and the average relative humidity during the experiment with the medium and fine sand was $20 \%$ and $17 \%$, respectively (unfortunately due to a technical problem, we could not continuously measure the ambient 
relative humidity during the experiment with the coarse sand, but we expect it to be in the same range and the differences have minimal impact on our results). The measured ambient temperature and relative humidity were obviously different from the outdoor values as the experiments were conducted in laboratory.

As mentioned earlier, the stage- 1 evaporation is controlled by the external conditions. The slight differences and fluctuations observed in the measured evaporation rates during stage-1 in our experiments are due to the fluctuation in air speed, ambient temperature and relative humidity. Also this implies that when extrapolating to the performance in the outdoors, the influence of higher air speeds would be to increase evaporation and further boost the effectiveness of the evaporative roofs. One can estimate this increase to be about $25 \%$ based on the relation between the Penman and Priestly-Taylor evaporation models, where a factor of 0.25 is used to model the contribution of the wind advection term.

According to Fig. 3(a), in all cases the slopes of the cumulative mass loss curves were initially high and equal (indicating a high evaporation rate which corresponds to the stage-1 evaporation). As mentioned earlier, during this period the drying process is dominated by the capillary induced liquid flow from the drying front (the interface between saturated and unsaturated zone) to the surface maintaining a high evaporation rate despite the receding drying front. As shown in Fig. 3, in all three cases investigated in the present work, the stage1 evaporation is followed by a transition period and stage- 2 evaporation. This figure clearly illustrates the effect of the texture of the porous media on the drying behaviour such that the medium with finer pores exhibits longer stage-1 evaporation. The relative evaporation rate in Fig. 3(b) was calculated by scaling the measured evaporation rate in each case by the potential evaporation rate measured from the container filled with water. 
Similar behaviour was observed in other studies $[24,25]$. Stage-1 evaporation ends when the upward capillary force transferring the liquid to the surface for vaporization is balanced by the downward gravity and viscous forces. The latter is negligible in sandy media due to the large pore sizes [24]. In porous media with finer pores such as clay, the upward capillary force maintains the hydraulic continuity over longer distances, prolonging the stage-1 evaporation (for more discussions about the effects of pore size distribution on the prolongation of stage-1 evaporation see Or et al. [30] and references therein). Fig. 3 illustrates the influence of the pore size on the dynamics of the transition from stage- 1 to stage-2 evaporation. The results show a sharper and shorter transition period as particle size (and probably the width and tale of the pore size distribution) decreases.

Different drying dynamics as influenced by the particle size distribution reflect different liquid phase distribution through the drying porous media. This will influence the temperature distribution as well as heat flux through the drying porous media, as will be discussed in the next section.

\subsection{Thermal signatures during drying of porous media}

Fig. 4(a) presents the recorded radiation flux by the pyranometer located in front of the bare roof in each round of the experiment and Fig. 4(b) shows the radiation flux recorded by the pyranometer placed in front of the container packed with sand grains scaled by the radiation flux received by the bare roof. The recorded radiation flux is not similar to the values typically observed in outdoor spaces. In our experiments the radiative flux was limited by the capacity of the metal halide lamps used in our experiments. However this difference in the radiative fluxes does not influence the process and physics explained in this paper. Although slight differences were observed between the radiation flux toward the bare roof in each round of the experiment (due to the slightly different environmental conditions), the scaled 
radiation flux in Fig. 4(b) is very close to 1 in all cases. This indicates an equal amount of radiation to the two containers used in each round of the experiment and enables us to accurately evaluate the effects of the presence of a drying porous medium together with the effect of its particle size distribution on the roof surface temperature.
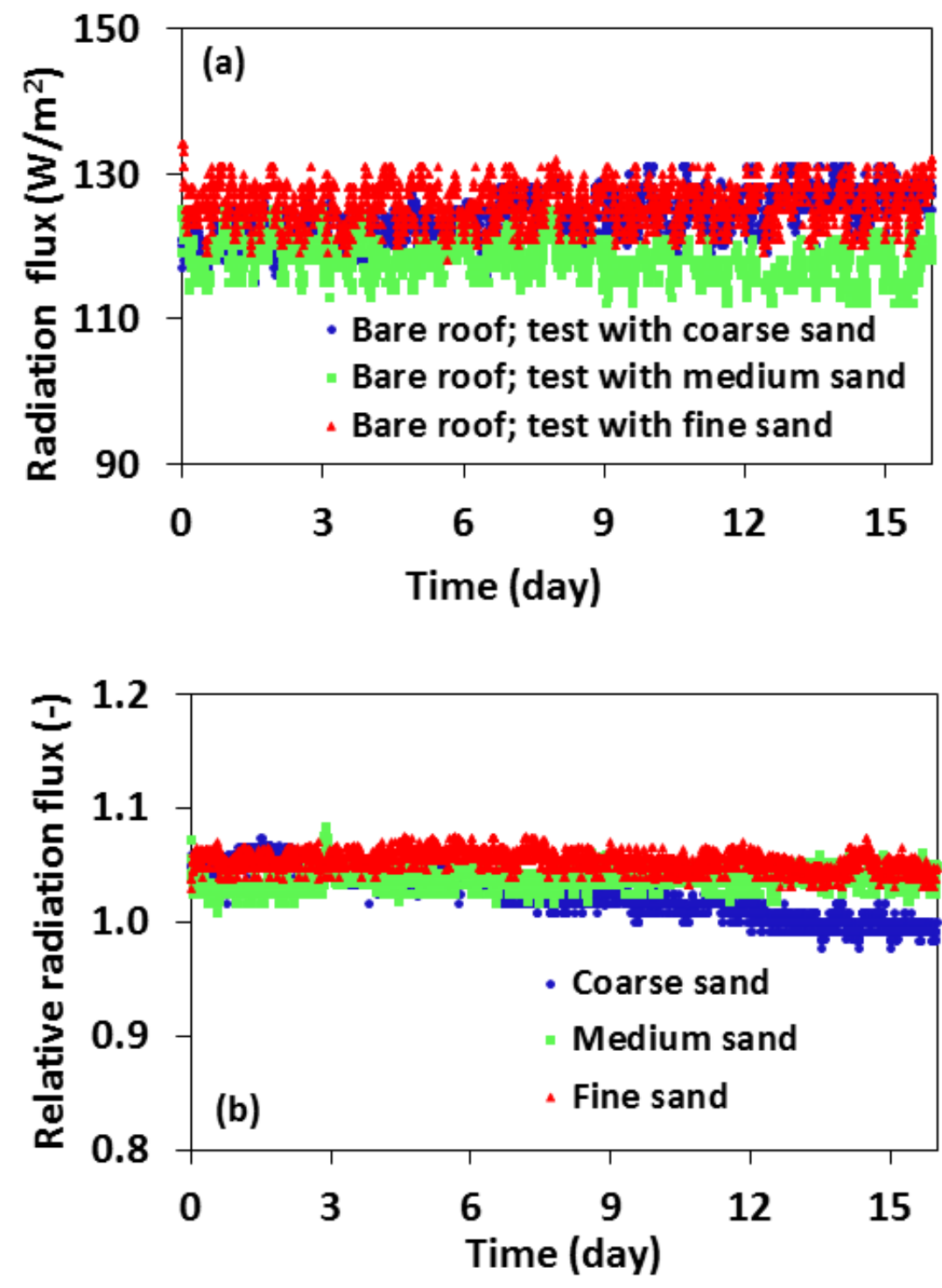

Fig. 4. (a) Radiation flux toward the bare roofs measured by the pyranometers during the experiments with the coarse, medium and fine sand as illustrated in the legend. (b) The radiation flux toward the roof filled with sand particles scaled by the radiation flux received by the bare roof. The closeness of the value of this relative 
radiation flux to 1 indicates equal radiation toward the two containers (i.e. bare roof and the one filled with drying porous media).

Although the radiation flux in each round of the experiment was relatively constant (as shown in Fig. 4), the ambient temperature, air speed, relative humidity, and also potentially ambient radiation, were not constant in our experiments, causing some fluctuations in the recorded bare roof temperature by the thermocouple as illustrated in Fig. 5. The attribution of the fluctuations to environmental conditions is supported by the clear diurnal cycle in the temperature plots.

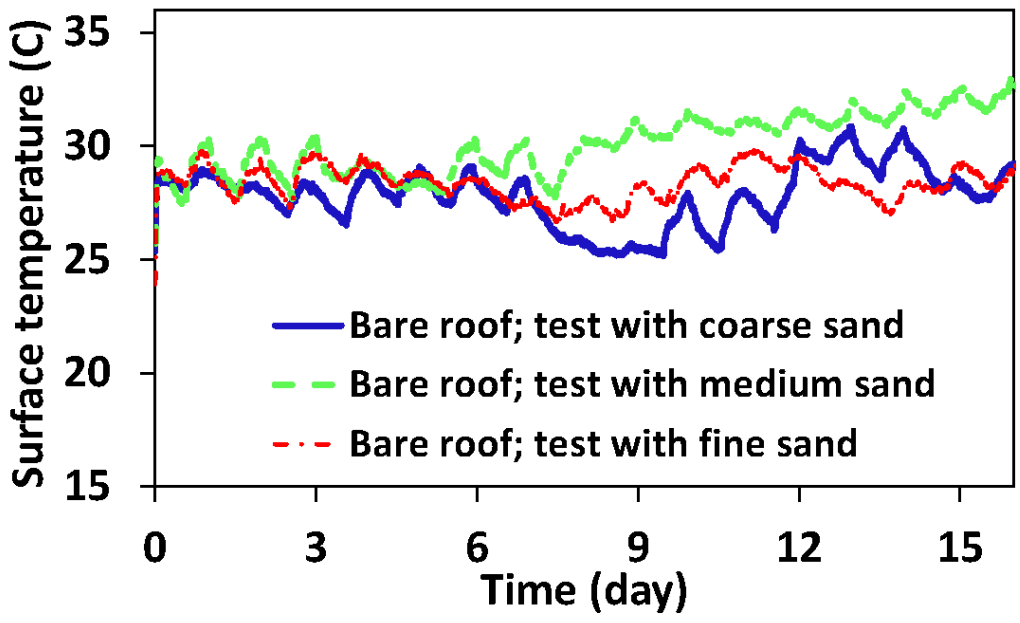

Fig. 5. Evolution of the surface temperature of the bare roof measured during the experiments with coarse, medium and fine sand. The observed temperature fluctuation is due to the fluctuation of the ambient conditions.

The temperature of the bare roof in each round of the experiment served as a reference to evaluate the performance of the drying porous media with a given particle size distribution in moderating the surface temperature. In other words, the measured temperature profiles through the drying porous media are scaled by the recorded temperature of the bare roof in each round of the experiment, enabling us to evaluate the influence of the particle size on the performance of the drying porous media. 
The temperature profiles through each drying container were different due to the different drying behaviour of the porous media as influenced by the particle size distribution. Fig. 6 illustrates the spatial and temporal distribution of temperature at the surface of the drying porous media during evaporation under the same cumulative evaporative mass losses (hence at different times for the different experiments).

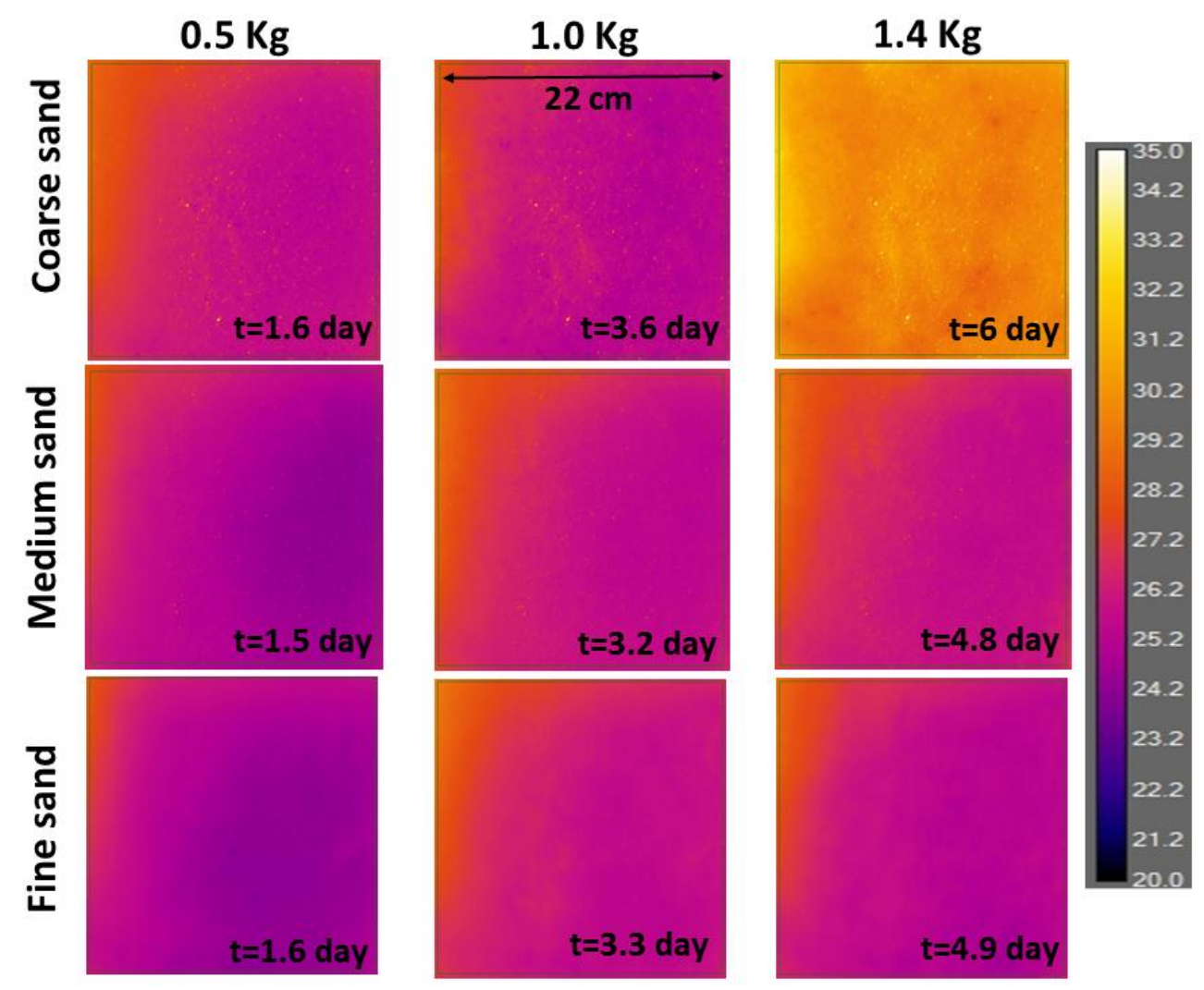

Fig. 6. Surface temperature of the coarse (top row), medium (middle row) and fine sand (bottom row) at different times from the onset of the experiments. The numbers at the top indicate the evaporative mass losses. The colour map indicates the temperature $\left({ }^{\circ} \mathrm{C}\right)$. Results show that after the same amount of mass loss from the coarse, medium and fine sand $(1.4 \mathrm{Kg})$, the surface of the coarse sand is warmer due to the earlier transition from stage-1 to stage-2 evaporation (see Fig. 3).

Fig. 6 shows that the surface temperature is higher as the particle size increases as a result of the earlier transition from stage- 1 to stage- 2 of evaporation. In other words, as particle size increases, the capillary length sustaining the hydraulic connections between the receding 
drying front and the surface (where evaporation occurs during stage-1) shortens, which results in an earlier disconnection of the hydraulic pathways between the saturated zone at the bottom and the surface. When the liquid connections are disrupted, the evaporation surface dries out resulting in higher temperatures, and this disconnection happens earlier in coarser sand both in terms of time and in terms of accumulated evaporative loss. This is why under the same cumulative evaporative water losses, the surface temperature of the container packed with coarser sand is higher as illustrated in Fig. 6.

Fig. 7 shows the evolution of the temperature at top and bottom of the container packed with the drying porous media together with the associated temperature of the bare roof. 

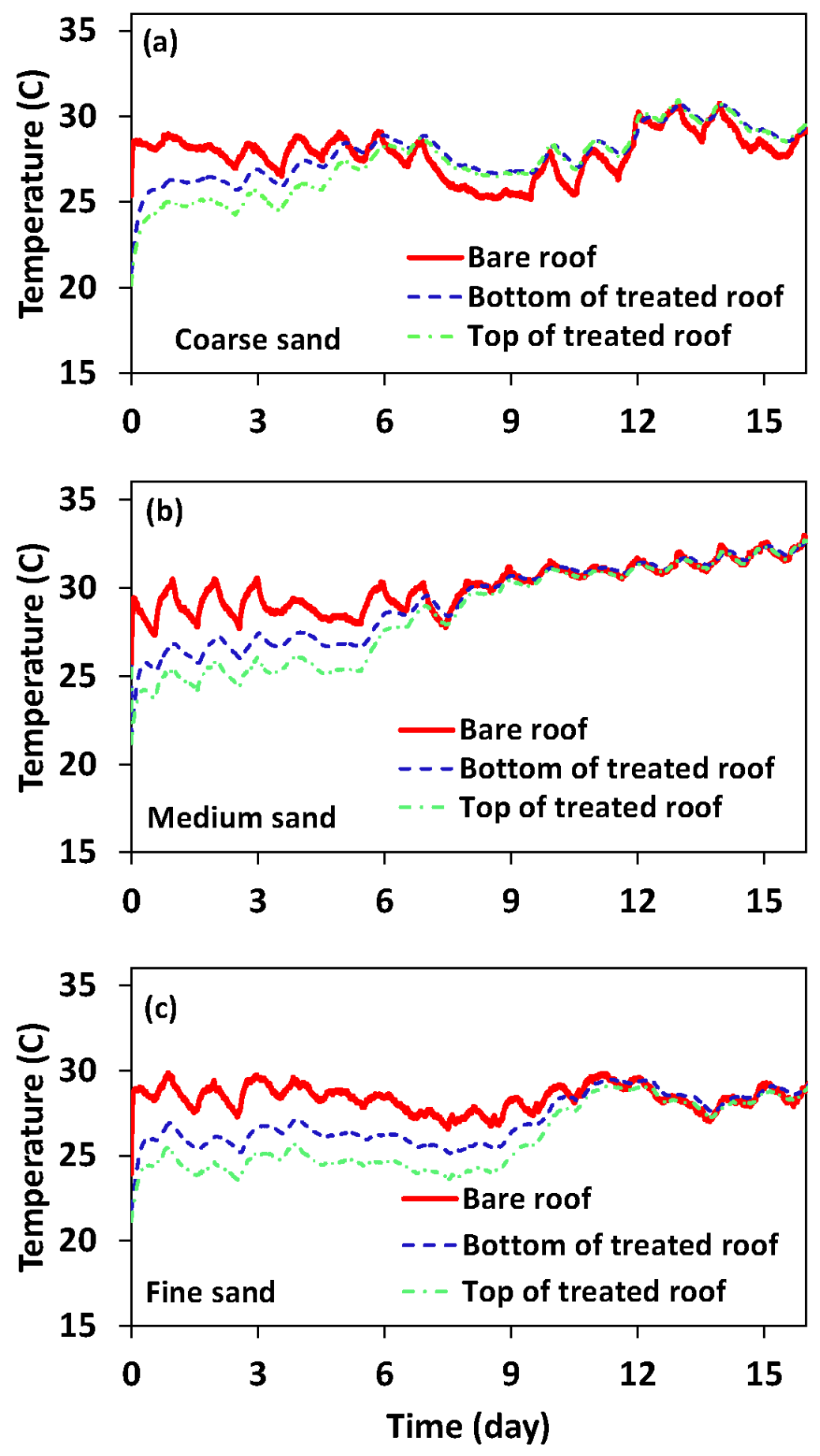

Fig. 7. The measured temperature at top and bottom of the container packed with drying coarse (a), medium (b) and fine (c) sand referred to as "treated roof" in the legend. Also presented is the measured temperature at the surface of the bare roof (i.e. the container without porous media).

The results show that in all cases, the temperature at the roof underlying drying sand packs is less than the bare roof up to a certain time, after which the presence of the porous medium 
has a negligible effect on reducing the roof temperature. Additionally, Fig. 7 shows that the finer the particle size of the drying porous medium, the longer is the period over which the surface temperature is less compared to the bare roof. This result can be interpreted in the light of the drying curves shown earlier.

Another interesting feature to note in Fig. 7 is that for the evaporative roofs the temperature at the top of the roof is lower than the temperature at the bottom. This indicates that evaporation in this case in fact manages not only to reduce the inward heat flux, but also to reverse the flux such that the roof exterior would be cooling the building. An indication of this cooling is that the temperature at the bottom is slightly lower than the measured room air temperature (which varied from about 28 to $30^{\circ} \mathrm{C}$ ); the bottom surface does not receive radiation and any deviation of its temperature from the ambient air would be related to the heat flux through the roof. A temperature lower than the ambient air at that surface indicates it is being cooled from above. This is not surprising in view of the low ambient relative humidity. Under such dry conditions, the wet bulb temperature (which one can simply define as the minimum temperature that a surface can cool to due to evaporation from its surface into the ambient environment) is significantly lower than the ambient air temperature (for our experimental conditions the wet bulb temperature can be computed from the air temperature and relative humidity and is $\approx 15^{\circ} \mathrm{C}$ ). An evaporating surface can cool to temperatures below those of the air, even when the surface is being heated by incoming radiation. It is clear that these conditions prevail in our experiment, and this reveals the potential of evaporative roofs not only to mitigate cooling loads (reduce inward heat flux), but furthermore to act as passive building coolers (create an outward heat flux).

Fig. 8 shows the dimensionless temperature at the surface and bottom of the drying porous media together with the associated measured drying curves. 

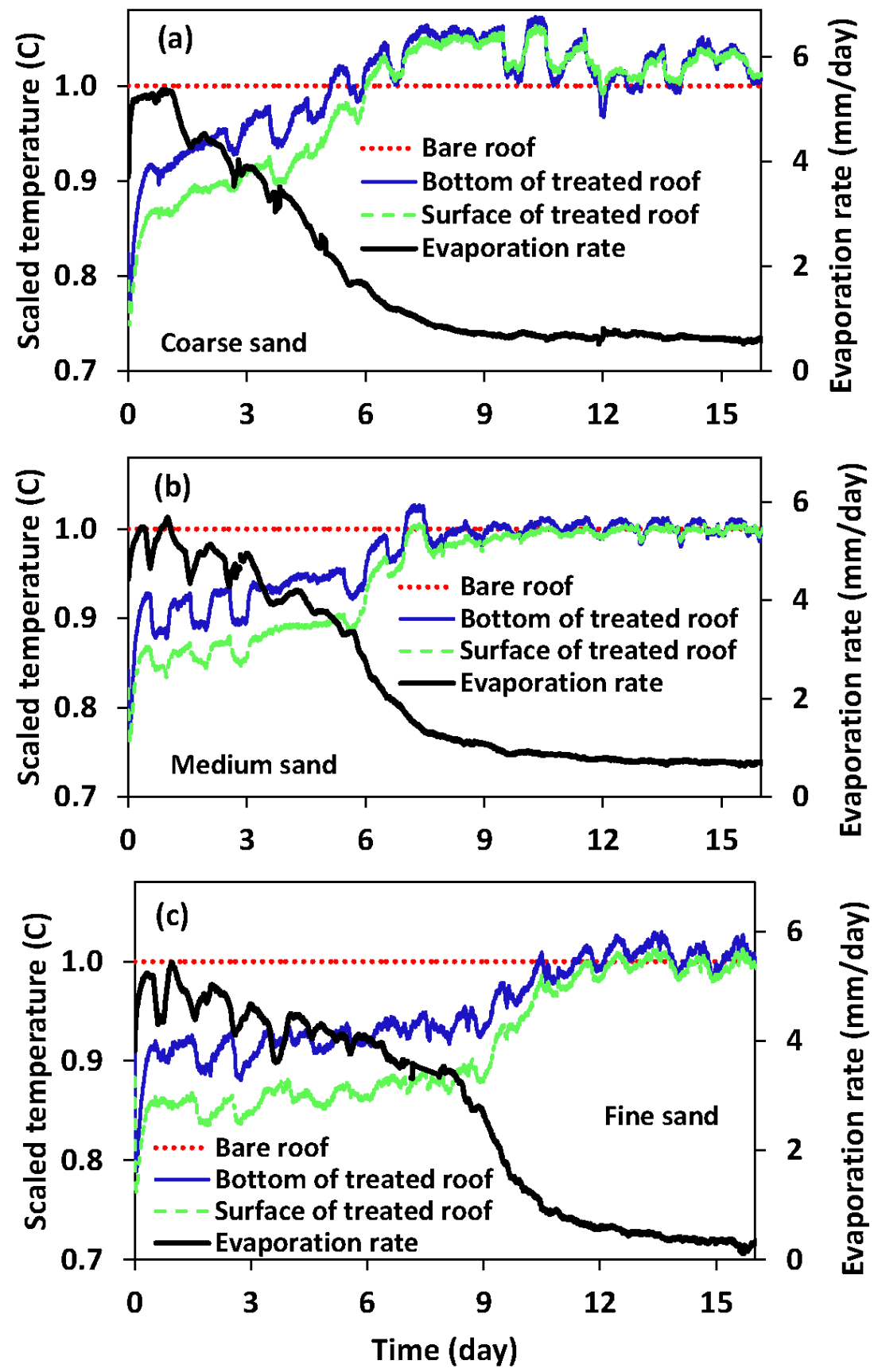

Fig. 8. Evolution of the temperature at the surface and bottom of the treated roof filled with coarse, medium and fine sand presented in (a), (b) and (c), respectively. The temperature was scaled by the temperature of the bare roof (using the values in ${ }^{\circ} \mathrm{C}$ ). Results show that the temperature of the treated roof (the roof filled with the drying porous media) is lower than the bare roof as long as the evaporation process remains during stage-1 evaporation. Due to the longer stage-1 evaporation in the case of fine sand, the effectiveness of the fine-textured material to moderate the roof surface temperature is improved. 
Fig. 8 shows that as long as the evaporation process is during stage-1, the temperature at the surface of the "treated roof", i.e. the roof with the overlying drying porous media, is lower than the bare roof. As discussed earlier and shown in previous studies [32], during stage-1 evaporation the surface remains partially wet. This reduces the temperature compared to the bare roof. Fig. 8 clearly shows that the end of stage- 1 evaporation marks the time when temperature begins to rise at the surface and bottom of the drying porous media due to the loss of liquid continuity with the surface, which eventually reduces the surface water content. This Fig. confirms the importance of the drying characteristics of the porous media on the performance of such a roofing system. An efficient and effective porous media for such a system should present a relatively long period of stage-1 evaporation, otherwise the influence of the presence of the porous medium will be minor.

Additionally, Fig. 8 shows that as the particle size decreases, the prolongation of stage-1 evaporation increases. Therefore, the application of a finer-textured medium will be more effective compared to the coarse-textured porous medium. It should be noted that in the range of the particle sizes used in our experiments, all three sandy media contain relatively large grains. If the drying porous medium were from a very fine-textured material, such as clay, the prolongation of stage-1 evaporation could be limited by viscous forces, which reduce the evaporation rate and also may cause desiccation cracking [24, 33]. However, these effects are negligible in our study. In any case, Fig. 8 shows that the application of drying porous media on the roof reduces the roof temperature by about $5{ }^{\circ} \mathrm{C}$ relative to the bare roof. Such a reduction has the potential to reduce cooling loads and carbon footprint, as well as energy bills bu requiring less energy to cool the building.

Moreover, Fig. 8 again shows that in all cases the temperature at the bottom of the drying porous media was higher than the one at the surface, which as discussed earlier illustrates the 
high potential of evaporative roofs to act as passive cooling systems. This potential is further exhibited in Fig. 9, which depicts the measured heat flux by the sensor buried in the middle of the drying porous media during each round of the experiment.

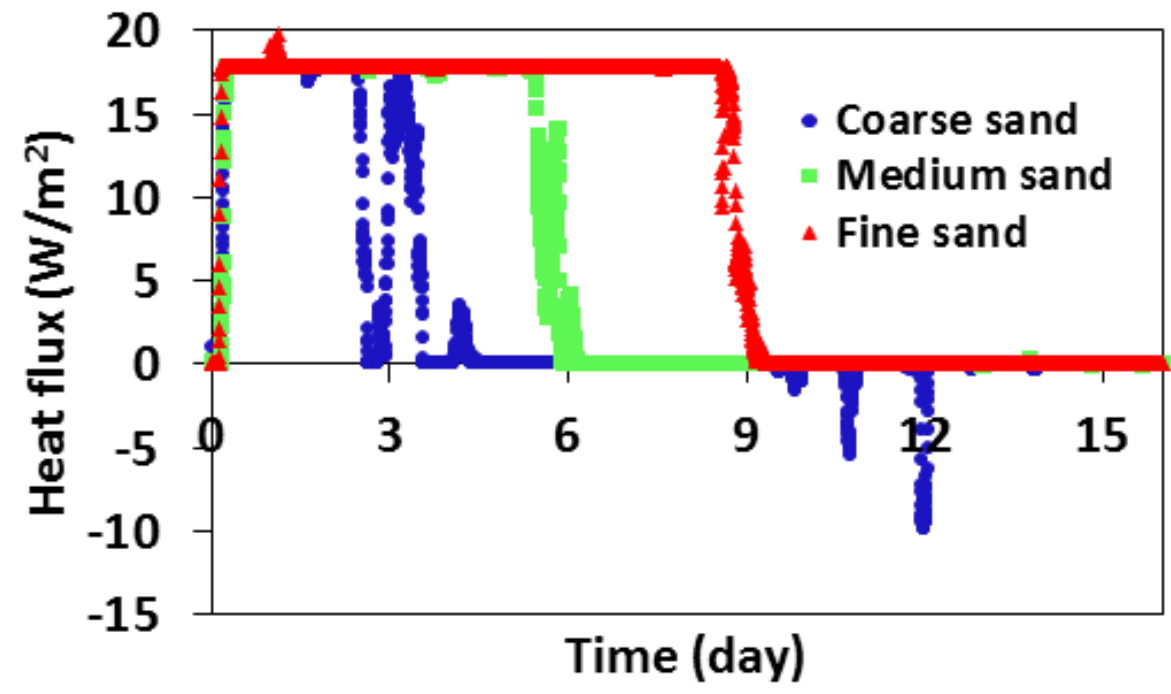

Fig. 9. The measured heat flux through the coarse, medium and fine sand. Positive heat flux indicates upward heat transfer.

In Fig. 9, positive values indicate upward heat flux (note that the absolute values reported in Fig. 9 might have been influenced by the threshold used in the working range of the heat flux sensor). Since the temperature at the bottom of the drying porous media was higher than the temperature at the surface during stage- 1 evaporation in all experiments conducted in this study (Fig. 7 and 8), upward heat flux was observed in all cases during stage-1. Again this is related to the low relative humidity that results in low wet bulb temperature and stronger evaporative cooling at the top surface to temperatures below the ambient air. If the roof surface is below the outdoor ambient air temperature and below the indoor desirable temperatures, the evaporation latent energy would be contributed to by the upward heat flux in the roofs as well as by a negative convective heat flux from the outdoor air towards the roof surface. 
This shows the potential of such a method (i.e. adding drying porous media above the roof to moderate roof surface temperature) to reduce the roof temperatures, the building cooling loads, and the convective heat flux to the exterior air that causes the urban heat island effect, potentially cooling the city $[34,35]$ for analyses of the influence of surface wetness and evaporation on the outdoor urban microclimate). However, since the temperature difference between top and bottom of the drying porous media reduces at the end of stage- 1 evaporation, the heat flux (driven by the temperature gradient) approaches to values close to zero and the cooling effect of the roof would become insignificant in the absence of rainfall or irrigation.

Combining the results presented in Fig. 7, 8 and 9, it can be concluded that (i) the effectiveness of applying porous media can be improved if the stage-1 evaporation is extended over a long period because it keeps the roof surface temperature lower for a longer time and may induce upward heat flux, and (ii) strong evaporation can very effectively cool the roof surface and provide benefits in terms of building energy consumption and the local microclimate. These are important considerations in designing evaporative roofs with porous media for real world applications.

\section{Summary and conclusions}

We have conducted carefully-controlled experiments to evaluate the application of drying porous media to reduce the roof surface temperature, with a focus on the design considerations that would control the performance of such roofs. The results showed the ability of porous materials to regulate the roof surface temperature, which eventually influences the building energy efficiency via reducing energy consumptions for cooling of buildings. Reducing the surface temperature would also have positive implications for mitigating urban heat islands since it would reduce the convective heat flux from the roof surface to the outdoor air. Several factors including fluctuating temperature and humidity, 
radiation, wind velocity, rain fall, structure of porous media, wettability of the grains, and the physical and chemical properties of the evaporating fluid influence the drying behaviour of porous media. In this paper, we investigated how the particle size distribution of a layer of porous medium affects its drying behaviour and thermal performance. Our results showed that finer-textured porous media are more appropriate for such an application because of the prolonged stage- 1 evaporation due to the efficient capillary flow connecting the wet zone at the bottom of the drying system to the surface. The thermal images, together with the data recorded by the thermocouples, established the direct connection between the drying behaviour, the recorded temperature profiles and the limit of the efficiency of the drying porous media. The end of stage- 1 evaporation coincides with the time when temperature at the treated roof begins to rise making the influence of the presence of the drying porous media negligible. This result shows the need to design porous materials that result in longer stage-1 evaporation if this method is to be used to reduce roof surface temperature. Application of the drying porous media lowered the irradiated laboratory surface temperature by about $5{ }^{\circ} \mathrm{C}$, but reductions on real roofs where the incoming radiation is about 10 times higher than in our laboratory experiments will be greater. The experiments also illustrated that evaporation can cool the surface to temperatures below ambient air (the theoretical limit would be the wet bulb temperature), thus reversing the heat flux and effectively extracting heat from the roof (not only reducing heat influx to the roof).

Although this study is still at an early stage, it clearly illustrates that this method has a potential to contribute toward reducing the energy consumption by buildings especially in places with hot climates. Further investigations are underway to evaluate the effects of the evaporating fluids (specifically saline water), as well as the heterogeneity of porous media on the efficiency of this method. More work is also needed, and is currently underway, to assess 
the performance of such roofs under real world conditions. To that end, we will implement a model of the evaporative roofs in the Princeton ROof Model (PROM, [16, 17]) and the Princeton Urban Canopy Model (PUCM, [29]), run PROM under the same experimental conditions we tested in this paper to validate our methods, and then apply the model under a wide range of real-world condition to assess the potential of evaporative roofs.

The development and use of evaporative roofs would significantly contribute towards the reduction of energy as well as water use in cities (since we could use non-fresh water), weaken the coupling of economic development and resource use, and facilitate the emergence of more sustainable cities in the future

\section{Acknowledgement}

We gratefully acknowledge funding by the The Leverhulme Trust to support this research (RPG-2014-331). We would like to thank Mr. Craig Shore and Mr. Andrew Evans for their assistance with the experimental setup. Elie Bou-Zeid was funded by the US National Science Foundation's Sustainability Research Network Cooperative Agreement 1444758.

\section{References}

[1] T.R. Oke, Towards a prescription for the greater use of climatic principles in settlement planning, Energ Buildings 7 (1984) 1-10.

[2] e. Kalnay, E., M. Cai, Impact of urbanization and land-use change on climate, Nature 423 (2003) 528-531.

[3] D. Li, E. Bou-Zeid, Synergistic interactions between urban heat islands and heat waves: the impact in cities is larger than the sum of its parts, J. Appl. Meteor. Climatol. 52 (9) (2013) 2051-2064. 
[4] A. Mavrogianni, M. Davies, M. Batty, S.E. Belcher, S.I. Bohnenstengel, D. Carruthers, et al., The comfort, energy and health implications of London's urban heat island, Building Serv. Eng. Res. Technol., 32(1) (2011) 35-52.

[5] M. Kolokotroni, X. Ren, M. Davies, A. Mavrogianni, A., London's urban heat island: Impact on current and future energy consumption in office buildings, Energy and Buildings 47 (2012) 302-311.

[6] L. Frazer, Paving paradise, Environmental Health Perspectives 113 (2005) 457-462.

[7] T. Runsheng, Y. Etzion, E. Erell, Experimental studies on a novel roof pond configuration for the cooling of buildings, Renewable Energy 28 (2003), 1513-1522.

[8] M. Kolokotroni, B.L. Gowreesunker, R. Giridharan, Cool roof technology in London: An experimental and modelling study, Energy and Buildings 67(2013) 658-667.

[9] D.J. Sailor, A green roof model for building energy simulation programs, Energy and Buildings 40(2008) 1466-1478.

[10] H.F. Castleton, V. Stovin, S.B.M. Beck, J.B. Davison, Green roofs; building energy savings and the potential for retrofit, Energy and Buildings 42(2010) 1582-1591.

[11] D. Li, E. Bou-Zeid, M. Oppenheimer, The Effectiveness of Cool and Green Roofs as Urban Heat Island Mitigation Strategies, Environ. Res. Lett. 9 (2014) 055002.

[12] S. Wanphen, K. Nagano, K. Experimental study of the performance of porous materials to moderate the roof surface temperature by its evaporative cooling effect, Building and Environment 44(2009) 338-351. 
[13] R. Crawford, A.K. da Silva, A.K., Experimental testing of a passive, evaporation-based roof cooling system, Energy and Buildings 71(2014) 12-19.

[14] S.B. Sadineni, S. Madala, R.F. Boehm, Passive building energy savings: A review of building envelope components, Renewable and Sustainable Energy Reviews 15(2011) 36173631.

[15] D. Jain, Modeling of solar passive techniques for roof cooling in arid regions, Building and Environment 41(2006), 277-287.

[16] T. Sun, E. Bou-Zeid, G.H. Ni, To irrigate or not to irrigate: Analysis of green roof performance via a vertically-resolved hygrothermal model, Building and Environment, 73(2013) 127-137.

[17] T. Sun, E. Bou-Zeid, Z.H. Wang, E. Zerba, G.H. Ni, Hydrometeorological determinants of green roof performance via a vertically-resolved model for heat and water transport, Building and Environment 60(2013) 211-224.

[18] G.W. Scherer, Theory of drying, J. Am. Ceram. Soc. 73(1990) 3-14.

[19] A.G. Yiotis, I.N. Tsimpanogiannis, A.K. Stubos, Y.C. Yortsos, Pore-networkstudy of the characteristic periods in the drying of porous materials, J. Colloid Interface Sci. 297(2006) $738-748$.

[20] K.M. Smits, V.V. Ngo, A. Cihan, T. Sakaki, T.H. Illangasekare, An evaluation of models of bare soil evaporation formulated with different land surface boundary conditions and assumptions, Water Resour. Res. 48(2012) W12526. 
[21] N. Shokri, D. Or, Drying patterns of porous media containing wettability contrasts, J. Colloid Interface Sci. 391(2013) 135-141.

[22] K.F. DeCarlo, N. Shokri, Salinity effects on cracking morphology and dynamics in 3-D desiccating clays, Water Resour. Res. 50(2014) 3052-3072.

[23] N. Shokri, M. Sahimi, D. Or, Morphology, Propagation Dynamics and Scaling Characteristics of Drying Fronts in Porous Media, Geophys. Res. Lett. 39(2012) L09401.

[24] P. Lehmann, S. Assouline, D. Or, Characteristic lengths affecting evaporative drying of porous media, Phys. Rev. E 77(2008) 056309.

[25] N. Shokri, D. Or, What determines drying rates at the onset of diffusion controlled stage2 evaporation from porous media?, Water Resour. Res. 47(2011) W09513.

[26] A.G. Yiotis, I.N. Tsimpanogiannis, A.K. Stubos, Y.C. Yortsos, Coupling between external and internal mass transfer during drying of a porous medium, Water Resour. Res. 43(2007) W06403.

[27] N. Shokri, P. Lehmann, P. Vontobel, D. Or, Drying front and water content dynamics during evaporation from sand delineated by neutron radiography, Water Resour. Res. 44(2008) W06418.

[28] M. Aminzadeh, D. Or, Energy partitioning dynamics of drying terrestrial surfaces, J Hydrol. 519(2014) 1257-1270.

[29] Z. Wang, E. Bou-Zeid, J.A. Smith, A coupled energy transport and hydrological model for urban canopies evaluated using a wireless sensor network, Quarterly Journal of the Royal Meteorological Society 139 (675) (2013) 1643-1657. 
[30] N. Grapsas, N. Shokri, Acoustic characteristics of fluid interface displacement in drying porous media, Int. J. Multiphas Flow 62(2014) 30-36.

[31] N. Shokri, G. Salvucci, Evaporation from porous media in the presence of a water table, Vadose Zone J. 10 (2011), 1309-1318.

[32] N. Shokri, Pore-scale dynamics of salt transport and distribution in drying porous media, Phys. Fluids 26(2014) 012106.

[33] K.F. DeCarlo, N. Shokri, Effects of substrate on cracking patterns and dynamics in desiccating clay layers, Water Resour. Res. 50(2014) 3039-3051.

[34] P. Ramamurthy, E. Bou-Zeid, Contribution of Impervious Surfaces to Urban Evaporation, Water Resour. Res 50(4) (2014) 2889-2902.

[35] P. Ramamurthy, E. Bou-Zeid, J.A. Smith, Z. Wang, M.L. Baeck, N.Z. Saliendra, J. Hom, C. Welty, Influence of Sub-Facet Heterogeneity and Material Properties on the Urban Surface Energy Budget J., Appl. Meteor. Climatol. 53(9)(2014) 2114-2129. 